\title{
Análisis químico de cerámicas calcolíticas de la Cuenca Media del Arlanzón (Burgos): un medio para la valoración de la procedencia y el intercambio
}

\author{
E. CARMONA BALLESTERO', S. PALMERO DÍAZ², M.A. ARNAIZ ALONSO' \\ 'Área de Arqueología. Facultad de Humanidades y Educación \\ 22Área de Química Analítica. Facultad de Ciencias Universidad de Burgos
}

\begin{abstract}
El trabajo presenta un estudio realizado sobre las cerámicas recuperadas en contextos calcolíticos ubicados en la Cuenca Media del Arlanzón. Dentro de los conjuntos de este tipo se reconocen tanto similitudes como discrepancias morfo-tipológicas y técnicas que plantean un problema arqueológico cuyo alcance ha de ser contrastado mediante otras metodologías distintas a la tipología. Como medio se ha utilizado el análisis químico de cerámicas, el cual permite observaciones menos subjetivas. Los resultados muestran aspectos interesantes que permiten discutir sobre la posible procedencia y los intercambios de las cerámicas calcolíticas en este sector de la Meseta Norte. Estos datos muestran una procedencia mayoritariamente local de las cerámicas junto a elementos intercambiados. En términos arqueológicos, el patrón se puede relacionar con un modelo doméstico de producción.
\end{abstract}

Palabras clave: Cerámica prehistórica, análisis químico de cerámica, procedencia e intercambio, Calcolítico, Meseta Norte.

Chemical analysis of chalcolithic pottery from Arlanzón river middle basin (Burgos): a way to examine the origin and exchange

The study is aimed on pottery that it is recovered in Chalcolithic contexts from Arlanzón River Middle Basin. Within these sets of pottery there are similarities and differences - morpho-typological and technical一, which it is necessary to study in order to understand its significance in archaeological terms. To doing so, we need to use a different method than typology. In fact, we used chemical analysis of ceramic as a way to obtain less subjective observations. The results allow discussing about origin and exchange of Chalcolithic pottery in this zone of the Spanish North Plateau. Data show a mainly local origin of ceramics with some exchanged artifacts. In archaeological terms, the pattern may be related to a domestic model of production.

Keywords: Prehistoric pottery, chemical analysis of ceramic, origin and exchange, Chalcolithic, Spanish North Plateau.

\section{INTRODUCCIÓN}

El análisis químico de pastas es una herramienta cada vez más empleada en el estudio arqueológico de la cerámica. Su empleo proporciona nuevas dimensiones que amplíanel alcance de los estudios tecno-tipológicos. Para ello existen distintas técnicas que permiten la reducción a lenguaje estadístico de esta composición, lo que posibilita una comparación menos subjetiva, en la que entran en juego elementos imperceptibles a través de un análisis macroscópico.

Esta comparación permite de momento, más que reconocer el origen de las pastas, identificar grupos con unas características químicas semejantes, que conforman familias, dentro de espacios o entornos geológicos determinados. En algunos casos también se reconocen elementos extraños dentro de un escenario concreto cuya composición química se aproximaría más a la de otros lugares. En este sentido, el fin del análisis ha sido dilucidar las características químicas de las cerámicas calcolíticas de la Cuenca Media del Arlanzón, comprobar su similitud o discordancia y contrastar esta información con la procedente de los estudios tipológicos y decorativos. Por esta razón se han muestreado un conjunto de piezas calcolíticas provenientes de distintos yacimientos de este marco geográfico (figura 1).

\section{PROBLEMÁTICA}

El estudio realizado se integra dentro de una investigación de mayor alcance orientada al conocimiento de las comunidades campesinas de la Prehistoria reciente en la zona nororiental de la Meseta Norte. En este sentido, el trabajo ha tenido en consideración únicamente los datos relacionados con el Calcolítico; es decir, solo parte de los análisis: 99 de los 185 realizados dentro del marco de investigación señalado. Durante esta fase, desde el punto de vista morfotipológico se reconocen dos conjuntos que se suceden en el tiempo: precampaniforme y campaniforme (figura 2). Cada 


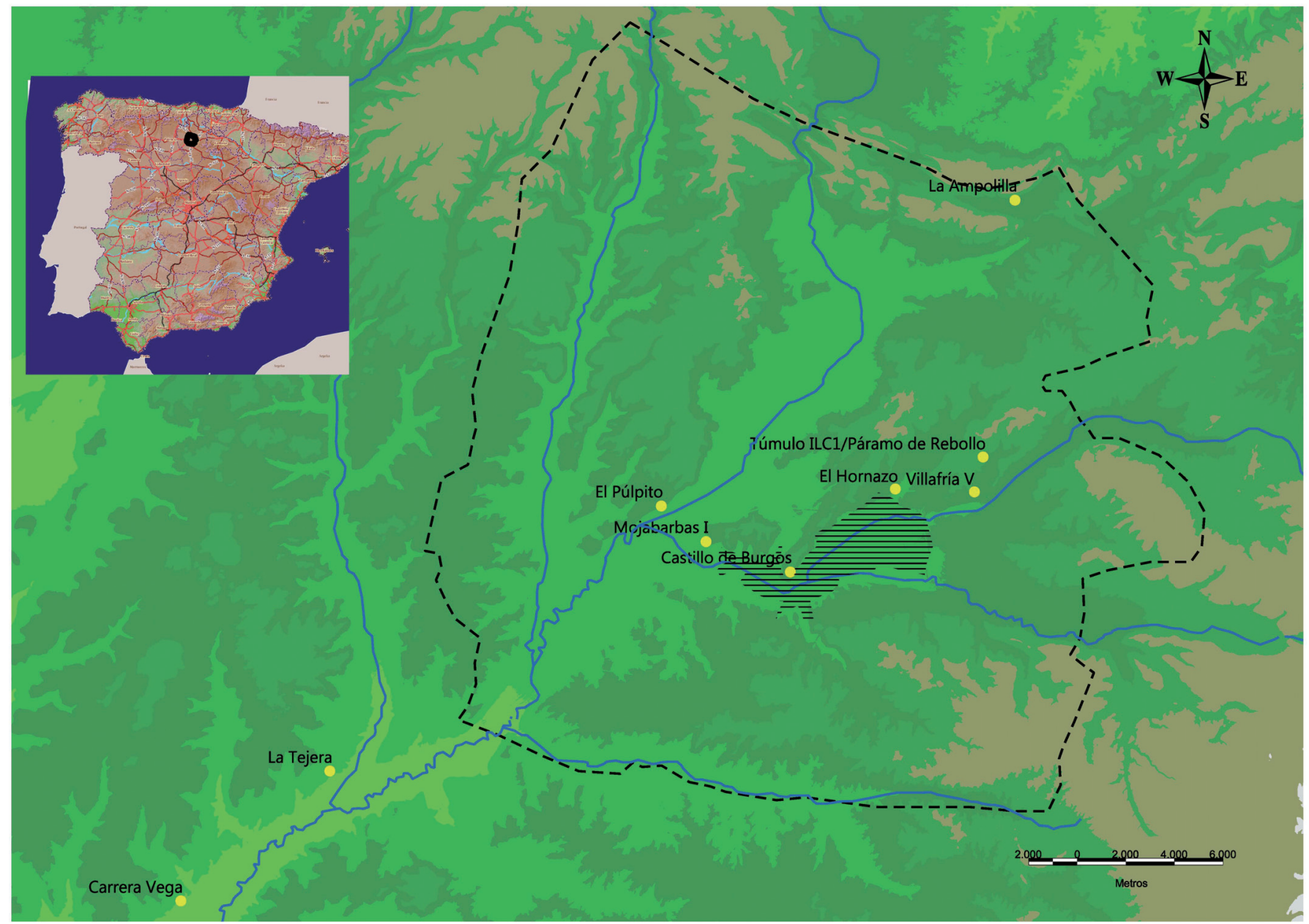

Figura 1. Localización de los yacimientos arqueológicos muestreados

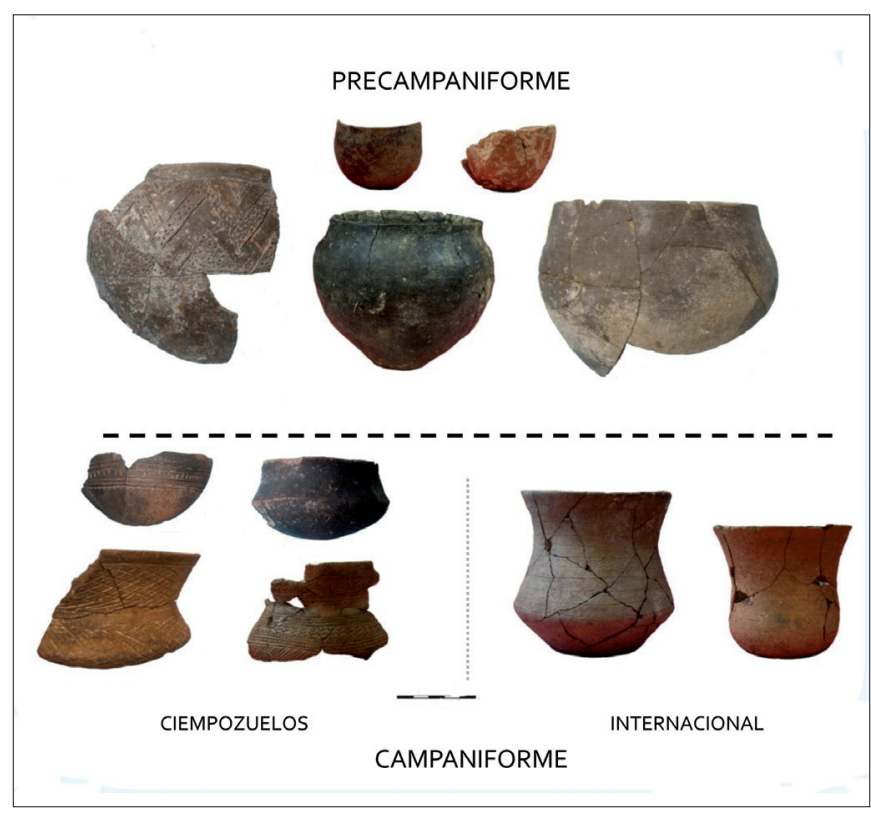

Figura 2. Cerámicas representativas de los conjuntos tipológicos del Calcolítico en la Cuenca Media del Arlanzón. Escala gráfica, $10 \mathrm{~cm}$.

uno tiene unos elementos formales definidos y particulares, que se podrán considerar propios de la Cuenca Media del Arlanzón. No obstante, las observaciones tipológicas hechas hasta el momento también dan cuenta de piezas con atributos extraños a este marco geográfico, sobre todo en contextos campaniformes. Es necesario dilucidar si esta observación tiene una correspondencia con los datos proporcionados por el análisis químico de cerámicas. En este sentido, la comparación pretende proporcionar un marco de discusión sobre la relación entre grupos establecidos químicamente y las evidencias arqueológicas.

\section{METODOLOGÍA}

La toma de muestras estuvo basada en experiencias previas (1). Consistió en la recopilación de un conjunto de casos representativos de cada yacimiento (entre 5 y 10 muestras en casi todos los sitios), afectando a recipientes incluidos en contextos distintos, y con atributos técnicos y decorativos diversos, como se puede apreciar en la tabla 1. Se ha intentado evitar un sesgo habitual en este tipo de estudios que se relaciona con el muestreo de las cerámicas que por alguna razón se consideran especiales, integrando piezas realmente representativas de la "normalidad" tanto técnica (cocciones, inclusiones y tratamientos diversos) como estilística (recipientes decorados con motivos habituales, extraños y piezas lisas). Para la determinación de los atributos de las cerámicas se ha seguido la metodología habitualmente empleada por el equipo de investigación del Área de Arqueología de la Universidad de Burgos (1-4). Esta se basa en la observación 
macroscópica y con lupa binocular de las piezas. Los criterios de clasificación siguen los patrones de Orton, Tyers y Vince (5), que son ampliamente admitidos y empleados (6-8) dentro de análisis arqueológicos de cerámica.
El conjunto de muestras definitivo está compuesto por 99 fracciones de pasta cerámica proveniente de 9 yacimientos diferentes de la Cuenca Media del Arlanzón (tabla 1): El Hornazo (EH), El Púlpito (EP), Castillo de Burgos (CB),

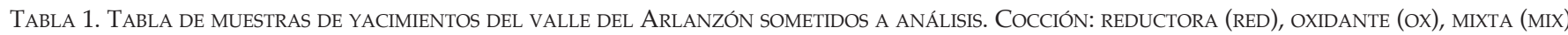
E IRREGULAR (IRR); TIPO DE INCLUSIÓN: CUARCÍTICAS (S), CUARZO (Q), CALIZA (L) Y MICA (M); FRECUENCIA DE LAS INCLUSIONES: ABUNDANTE (AB), MODERADA (MO) Y ESCASA (ES); TAMAÑo DE LAS INCLUSIONES: FinO (F), MEDiO (M) Y GRUESO (G); AtRIBUCión CULTURAL: CALCOLÍTICO PRECAMPANIFORME (CPR) Y Calcolítico Campaniforme (CC).

\begin{tabular}{|c|c|c|c|c|c|c|c|c|c|c|}
\hline Muestra & Yacimiento & Contexto & UE & Sigla & Cocción & Tipo & Frecuencia & Tamaño & $\begin{array}{c}\text { Adscripción } \\
\text { Cultural }\end{array}$ & Observaciones \\
\hline 1 & $\mathrm{EH}$ & 3 & 41 & $04.23 / 41.78$ & irr & $\mathrm{S} / \mathrm{L}$ & $a b$ & $f / g$ & $\mathrm{CPr}$ & \\
\hline 2 & $\mathrm{EH}$ & 3 & 41 & $04.23 / 41.124$ & irr & $\mathrm{S} / \mathrm{L}$ & $a b$ & $f / g$ & $\mathrm{CPr}$ & \\
\hline 3 & $\mathrm{EH}$ & 3 & 41 & $04.23 / 41.142$ & irr & $\mathrm{S} / \mathrm{L}$ & $a b$ & $f / g$ & $\mathrm{CPr}$ & \\
\hline 4 & $\mathrm{EH}$ & 3 & 41 & $04.23 / 41.155$ & irr & $\mathrm{S} / \mathrm{L}$ & $a b$ & $\mathrm{f} / \mathrm{g}$ & $\mathrm{CPr}$ & \\
\hline 5 & $\mathrm{EH}$ & 3 & 41 & $04.23 / 41.165$ & irr & $\mathrm{S} / \mathrm{L}$ & $a b$ & $f / g$ & $\mathrm{CPr}$ & \\
\hline 6 & $\mathrm{EP}$ & 14 & 141 & $2009 / 38 / 141 /$ & irr & S & es & g & $\mathrm{CPr}$ & Datación $3880 \pm 25$ BP \\
\hline 7 & $\mathrm{EP}$ & 14 & 141 & $2009 / 38 / 141 /$ & ox & $\mathrm{L}$ & es & $\mathrm{g}$ & $\mathrm{CPr}$ & \\
\hline 8 & $\mathrm{EP}$ & 14 & 141 & $2009 / 38 / 141 /$ & $\operatorname{mix}$ & Q & es & $\mathrm{f}$ & $\mathrm{CPr}$ & \\
\hline 9 & $\mathrm{EP}$ & 14 & 141 & $2009 / 38 / 141 /$ & irr & $Q$ & es & $\mathrm{g}$ & $\mathrm{CPr}$ & \\
\hline 10 & $\mathrm{EP}$ & 14 & 141 & $2009 / 38 / 141 /$ & irr & Q & es & $\mathrm{g}$ & $\mathrm{CPr}$ & \\
\hline 48 & $\mathrm{EH}$ & 165 & 1651 & $04.23 / 1651.1$ & irr & $\mathrm{Q}$ & es & $\mathrm{g}$ & $\mathrm{CPr}$ & pastillas repujadas \\
\hline 49 & $\mathrm{EH}$ & 165 & 1651 & $04.23 / 1651.2$ & irr & Q & es & g & $\mathrm{CPr}$ & pastillas repujadas \\
\hline 50 & $\mathrm{EH}$ & 165 & 1651 & $04.23 / 1651.3$ & $\operatorname{mix}$ & S/Q & $a b$ & $\mathrm{f} / \mathrm{m}$ & $\mathrm{CPr}$ & perforaciones \\
\hline 51 & $\mathrm{EH}$ & 165 & 1651 & $04.23 / 1651.4$ & irr & $\mathrm{L}$ & mo & g & $\mathrm{CPr}$ & pastillas repujadas \\
\hline 52 & $\mathrm{EH}$ & 165 & 1651 & $04.23 / 1651.5$ & irr & $\mathrm{L}$ & es & $\mathrm{m}$ & $\mathrm{CPr}$ & pastillas repujadas \\
\hline 53 & $\mathrm{EH}$ & 165 & 1651 & $04.23 / 1651.6$ & irr & Q & mo & $\mathrm{m}$ & $\mathrm{CPr}$ & acanalada \\
\hline 54 & $\mathrm{EH}$ & 165 & 1651 & $04.23 / 1651.7$ & red & S/Q & $a b$ & $\mathrm{f} / \mathrm{m}$ & $\mathrm{CPr}$ & pastillas repujadas \\
\hline 55 & $\mathrm{EH}$ & 165 & 1651 & $04.23 / 1651.8$ & irr & $S / Q$ & $a b$ & $\mathrm{f} / \mathrm{m}$ & $\mathrm{CPr}$ & contínua \\
\hline 56 & $\mathrm{EH}$ & 165 & 1651 & $04.23 / 1651.9$ & irr & $\mathrm{L} / \mathrm{Q}$ & $a b$ & $\mathrm{~m}$ & $\mathrm{CPr}$ & perforaciones \\
\hline 57 & $\mathrm{EH}$ & 165 & 1651 & $04.23 / 1651.10$ & irr & $\mathrm{Q}$ & mo & $\mathrm{m}$ & $\mathrm{CPr}$ & \\
\hline 58 & $\mathrm{EH}$ & 165 & 1651 & $04.23 / 1651.11$ & $\operatorname{mix}$ & Q & mo & g & $\mathrm{CPr}$ & \\
\hline 59 & $\mathrm{EH}$ & 165 & 1651 & $04.23 / 1651.12$ & $\operatorname{mix}$ & Q & mo & g & $\mathrm{CPr}$ & \\
\hline 60 & $\mathrm{EH}$ & 165 & 1651 & $04.23 / 1651.13$ & red & S/Q & mo & $\mathrm{f} / \mathrm{m}$ & $\mathrm{CPr}$ & \\
\hline 61 & $\mathrm{EH}$ & 165 & 1651 & $04.23 / 1651.14$ & red & S/Q & mo & $f / g$ & $\mathrm{CPr}$ & \\
\hline 62 & $\mathrm{EH}$ & 165 & 1651 & $04.23 / 1651.15$ & $\operatorname{mix}$ & $S$ & $a b$ & $f / g$ & $\mathrm{CPr}$ & \\
\hline 63 & $\mathrm{EH}$ & 165 & 1651 & $04.23 / 1651.16$ & irr & S & mo & $\mathrm{f}$ & $\mathrm{CPr}$ & \\
\hline 64 & $\mathrm{EH}$ & 165 & 1651 & $04.23 / 1651.17$ & irr & Q & mo & $f / g$ & $\mathrm{CPr}$ & \\
\hline 65 & $\mathrm{EH}$ & 165 & 1651 & $04.23 / 1651.18$ & red & Q & $a b$ & g & $\mathrm{CPr}$ & \\
\hline 66 & $\mathrm{EH}$ & 165 & 1651 & $04.23 / 1651.19$ & irr & $\mathrm{Q}$ & mo & $\mathrm{m}$ & $\mathrm{CPr}$ & \\
\hline 67 & $\mathrm{EH}$ & 165 & 1651 & $04.23 / 1651.20$ & $\operatorname{mix}$ & S & mo & $\mathrm{f}$ & $\mathrm{CPr}$ & \\
\hline 68 & $\mathrm{EH}$ & 165 & 1651 & $04.23 / 1651.24$ & irr & $\mathrm{S} / \mathrm{Q}$ & $\mathrm{ab}$ & $f / g$ & $\mathrm{CPr}$ & contínua \\
\hline 69 & $\mathrm{EH}$ & 165 & 1651 & $04.23 / 1651.29$ & red & Q & $a b$ & g & $\mathrm{CPr}$ & pastillas repujadas \\
\hline 70 & $\mathrm{EH}$ & 165 & 1651 & $04.23 / 1651.31$ & $\operatorname{mix}$ & S/Q & mo & $f / g$ & $\mathrm{CPr}$ & puntos \\
\hline 71 & $\mathrm{EH}$ & 165 & 1651 & $04.23 / 1651.32$ & $\operatorname{mix}$ & S & $a b$ & $\mathrm{f}$ & $\mathrm{CPr}$ & puntos \\
\hline 72 & $\mathrm{EH}$ & 165 & 1651 & $04.23 / 1651.37$ & red & S/Q & $a b$ & $\mathrm{f}$ & $\mathrm{CPr}$ & contínua \\
\hline 73 & $\mathrm{CB}$ & $\begin{array}{c}\text { rellenos } \\
\text { medievales }\end{array}$ & $\mathrm{R}$ & $\mathrm{C} / \mathrm{SII} / \mathrm{R} / 2$ & $\operatorname{mix}$ & S/Q & $a b$ & $\mathrm{f}$ & $\mathrm{CC}$ & puntillado geométrico \\
\hline 74 & $\mathrm{CB}$ & palimpsesto & XIII & C/III/XIII/1012 & irr & $\mathrm{S} / \mathrm{L}$ & $\mathrm{ab}$ & $\mathrm{f}$ & $\mathrm{CC}$ & internacional \\
\hline 75 & $\mathrm{CB}$ & palimpsesto & XIII & C/III / XIII / 418 & irr & $\mathrm{L}$ & es & $\mathrm{m}$ & $\mathrm{CC}$ & puntillado geométrico \\
\hline 76 & $\mathrm{CB}$ & palimpsesto & XIII & C/III / XIII/ 563 & irr & S & $\mathrm{ab}$ & $\mathrm{f}$ & $\mathrm{CC}$ & CZM \\
\hline 77 & $\mathrm{CB}$ & palimpsesto & XIII & C/III/XIII/396 & $\operatorname{mix}$ & $\mathrm{L}$ & $a b$ & $\mathrm{~m}$ & $\mathrm{CC}$ & puntillado geométrico \\
\hline 78 & $\mathrm{CB}$ & palimpsesto & XIII & C/III/XIII/ 394 & $\operatorname{mix}$ & $S$ & mo & $\mathrm{f}$ & $\mathrm{CC}$ & puntillado geométrico \\
\hline 79 & $\mathrm{CB}$ & palimpsesto & XIII & C/III / XIII / 403 & $\operatorname{mix}$ & S & $a b$ & $\mathrm{f}$ & $\mathrm{CC}$ & CZM \\
\hline 80 & $\mathrm{CB}$ & $\begin{array}{c}\text { rellenos } \\
\text { medievales }\end{array}$ & $\mathrm{R}$ & $\mathrm{C} / \mathrm{SII} / \mathrm{R} / 1$ & $\operatorname{mix}$ & S & $a b$ & $\mathrm{f}$ & $\mathrm{CC}$ & cordado \\
\hline 81 & $\mathrm{CB}$ & palimpsesto & XIII & C/SII/XIII/ 66 & red & $\mathrm{Q}$ & mo & $\mathrm{m}$ & $\mathrm{CC}$ & puntillado geométrico \\
\hline 82 & ILC & $\begin{array}{c}\text { relleno } \\
\text { estructura }\end{array}$ & EII & ILC1/174/EII & $\operatorname{mix}$ & $\mathrm{S} / \mathrm{M}$ & $a b$ & $\mathrm{f}$ & $\mathrm{CC}$ & internacional \\
\hline 83 & ILC & $\begin{array}{l}\text { relleno } \\
\text { estructura }\end{array}$ & EII & ILC1/172/EII & $\operatorname{mix}$ & $\mathrm{S} / \mathrm{M} / \mathrm{Q}$ & $a b$ & $\mathrm{f}$ & $\mathrm{CC}$ & internacional \\
\hline
\end{tabular}




\begin{tabular}{|c|c|c|c|c|c|c|c|c|c|c|}
\hline Muestra & Yacimiento & Contexto & $\mathrm{UE}$ & Sigla & Cocción & Tipo & Frecuencia & Tamaño & $\begin{array}{l}\text { Adscripción } \\
\text { Cultural }\end{array}$ & Observaciones \\
\hline 84 & ILC & $\begin{array}{c}\text { relleno } \\
\text { estructura }\end{array}$ & EII & ILC1/126/EII & $\operatorname{mix}$ & S & es & $\mathrm{f}$ & $\mathrm{CC}$ & \\
\hline 85 & ILC & $\begin{array}{c}\text { relleno } \\
\text { estructura }\end{array}$ & EII & ILC1/227/EII & mix & L & $\mathrm{ab}$ & $\mathrm{m}$ & $\mathrm{CC}$ & \\
\hline 86 & ILC & $\begin{array}{l}\text { relleno } \\
\text { estructura }\end{array}$ & EII & ILC1/172/EII & $\operatorname{mix}$ & S & es & f & $\mathrm{CC}$ & \\
\hline 87 & $\mathrm{MB}$ & Superficie & sup & MBII $/ 221 / \mathrm{S}$ & $\operatorname{mix}$ & $\mathrm{s}$ & es & $f$ & $\mathrm{CC}$ & ciempozuelos \\
\hline 88 & MB & Superficie & sup & MBII /224/S & $\operatorname{mix}$ & $\mathrm{s}$ & es & $\mathrm{f}$ & $\mathrm{CC}$ & ciempozuelos \\
\hline 89 & MB & Superficie & sup & MBII $/ 220 / \mathrm{S}$ & $\operatorname{mix}$ & $\mathrm{s}$ & es & $\mathrm{f}$ & $\mathrm{CC}$ & ciempozuelos \\
\hline 90 & MB & Superficie & sup & MBII/215/S & $\operatorname{mix}$ & $\mathrm{S} / \mathrm{L}$ & es & $\mathrm{f}$ & $\mathrm{CC}$ & ciempozuelos \\
\hline 91 & MB & Superficie & sup & & mix & $\mathrm{L}$ & $\mathrm{ab}$ & $\mathrm{f} / \mathrm{m}$ & $\mathrm{CC}$ & mamelón \\
\hline 92 & $\mathrm{MB}$ & Superficie & sup & $\mathrm{MBII} / 130 / \mathrm{S}$ & irr & $\mathrm{L}$ & $\mathrm{ab}$ & $\mathrm{f} / \mathrm{m}$ & $\mathrm{CC}$ & \\
\hline 93 & MB & Superficie & sup & & $\operatorname{mix}$ & $\mathrm{s} / \mathrm{Q}$ & $a b$ & $\mathrm{f} / \mathrm{m}$ & $\mathrm{CC}$ & \\
\hline 95 & MB & Superficie & sup & & irr & $\mathrm{S} / \mathrm{L}$ & es & $\mathrm{f}$ & $\mathrm{CC}$ & ciempozuelos \\
\hline 96 & $\mathrm{MB}$ & Superficie & sup & & irr & $\mathrm{L}$ & mo & $\mathrm{m}$ & $\mathrm{CC}$ & liso \\
\hline 97 & VFV & cabaña & & $\mathrm{VFV} / 247$ & red & $\mathrm{s}$ & es & $\mathrm{f}$ & $\mathrm{CC}$ & valle ebro \\
\hline 98 & VFV & cabaña & & VFV/414 & mix & $\mathrm{s}$ & mo & $\mathrm{f}$ & $\mathrm{CC}$ & valle ebro \\
\hline 99 & VFV & cabaña & & $\mathrm{VFV} / 230$ & irr & $\mathrm{s}$ & $\mathrm{ab}$ & $f$ & $\mathrm{CC}$ & valle ebro \\
\hline 100 & VFV & cabaña & & $\mathrm{VFV} / 248$ & red & S & $\mathrm{ab}$ & $\mathrm{f}$ & $\mathrm{CC}$ & valle ebro \\
\hline 101 & VFV & cabaña & & $\mathrm{VFV} / 231$ & ox & $\mathrm{s}$ & $\mathrm{ab}$ & $\mathrm{f}$ & $\mathrm{CC}$ & valle ebro \\
\hline 102 & VFV & cabaña & & VFV/389 & irr & $\mathrm{L}$ & es & $\mathrm{g}$ & $\mathrm{CC}$ & \\
\hline 103 & VFV & cabaña & & $\mathrm{VFV} / 245$ & $\operatorname{mix}$ & $\mathrm{s}$ & es & $\mathrm{f}$ & $\mathrm{CC}$ & ciempozuelos \\
\hline 104 & VFV & cabaña & & VFV/ 254 & $\operatorname{mix}$ & $\mathrm{L}$ & es & g & $\mathrm{CC}$ & \\
\hline 105 & VFV & cabaña & & $\mathrm{VFV} / 423$ & red & $\mathrm{s}$ & es & $\mathrm{f}$ & $\mathrm{CC}$ & contínua \\
\hline 106 & LA & Superficie & sup & $\mathrm{AI} / \mathrm{S} / 11$ & ox & $\mathrm{s}$ & $\mathrm{ab}$ & $\mathrm{f}$ & $\mathrm{CC}$ & ciempozuelos \\
\hline 107 & LA & Superficie & sup & $\mathrm{AI} / \mathrm{S} / 473$ & mix & $\mathrm{s}$ & $\mathrm{ab}$ & $\mathrm{f}$ & $\mathrm{CC}$ & ciempozuelos \\
\hline 108 & LA & Superficie & sup & $\mathrm{AI} / \mathrm{S} / 468$ & mix & $\mathrm{s}$ & mo & $\mathrm{f}$ & $\mathrm{CC}$ & ciempozuelos \\
\hline 109 & LA & Superficie & sup & $\mathrm{AI} / \mathrm{S} / 479$ & mix & $\mathrm{S}$ & mo & $\mathrm{f}$ & CC & ciempozuelos \\
\hline 110 & LA & Superficie & sup & $\mathrm{AI} / \mathrm{S} / 481$ & mix & $\mathrm{s}$ & es & $\mathrm{m}$ & $\mathrm{CC}$ & ciempozuelos \\
\hline 111 & LA & Superficie & sup & $\mathrm{AI} / \mathrm{S} / 83$ & irr & $\mathrm{s}$ & es & $\mathrm{f}$ & $\mathrm{CPr}$ & puntos \\
\hline 112 & LA & Superficie & sup & $\mathrm{AI} / \mathrm{S} / 71$ & red & $\mathrm{S} / \mathrm{Q}$ & es & $\mathrm{f} / \mathrm{m}$ & $\mathrm{CPr}$ & moldura \\
\hline 113 & LA & Superficie & sup & $\mathrm{AI} / \mathrm{S} / 560$ & irr & $\mathrm{L}$ & es & $\mathrm{g}$ & $\mathrm{CC}$ & ciempozuelos \\
\hline 114 & LA & Superficie & sup & $\mathrm{AI} / \mathrm{S} / 472$ & $\operatorname{mix}$ & $S$ & es & $\mathrm{f}$ & $\mathrm{CC}$ & ciempozuelos \\
\hline 115 & LA & Superficie & sup & $\mathrm{AI} / \mathrm{S} / 475$ & red & $\mathrm{s}$ & es & $\mathrm{f}$ & $\mathrm{CC}$ & ciempozuelos \\
\hline 116 & LT & Hoyo & 3 & $2010 / 19 / 3 / 3$ & mix & $\mathrm{S} / \mathrm{L}$ & mo & $\mathrm{f} / \mathrm{m}$ & $\mathrm{CC}$ & ciempozuelos \\
\hline 117 & LT & Hoyo & 3 & $2010 / 19 / 3 / 1$ & irr & $\mathrm{S} / \mathrm{L}$ & $\mathrm{mo}$ & $\mathrm{f} / \mathrm{m}$ & $\mathrm{CC}$ & liso \\
\hline 118 & LT & Hoyo & 3 & $2010 / 19 / 3 / 4$ & red & $\mathrm{s}$ & es & $\mathrm{m}$ & $\mathrm{CC}$ & ciempozuelos \\
\hline 119 & LT & Hoyo & 3 & $2010 / 19 / 3 / 7$ & red & $\mathrm{s}$ & $\mathrm{ab}$ & $\mathrm{f}$ & $\mathrm{CC}$ & ciempozuelos \\
\hline 120 & LT & Hoyo & 3 & $2010 / 19 / 3 / 12$ & mix & $\mathrm{s}$ & es & $\mathrm{f}$ & $\mathrm{CC}$ & ciempozuelos \\
\hline 121 & LT & Hoyo & 3 & 2010/19/3/13 & irr & $\mathrm{s}$ & $\mathrm{ab}$ & $\mathrm{f}$ & $\mathrm{CC}$ & \\
\hline 122 & LT & Hoyo & 3 & $2010 / 19 / 3 / 13$ & $\operatorname{mix}$ & $\mathrm{s}$ & es & $\mathrm{f}$ & $\mathrm{CC}$ & \\
\hline 123 & LT & Hoyo & 3 & 2010/19/3/13 & $\operatorname{mix}$ & $\mathrm{s}$ & es & $\mathrm{f}$ & $\mathrm{CC}$ & \\
\hline 124 & LT & Hoyo & 3 & $2010 / 19 / 3 / 13$ & ox & $\mathrm{L}$ & es & $\mathrm{m}$ & $\mathrm{CC}$ & \\
\hline 125 & LT & Hoyo & 3 & 2010/19/3/13 & irr & $\mathrm{L}$ & es & $\mathrm{m}$ & $\mathrm{CC}$ & \\
\hline 136 & $\mathrm{CV}$ & Hoyo & 601 & $2010 / 19 / 1 / 601 / 1$ & $\operatorname{mix}$ & $\mathrm{s}$ & es & $\mathrm{f}$ & $\mathrm{CC}$ & ciempozuelos \\
\hline 140 & $\mathrm{EH}$ & 139 & 1391 & $04.23 / 1391.37$ & irr & $\mathrm{Q}$ & es & $\mathrm{g}$ & $\mathrm{CPr}$ & pastillas repujadas. \\
\hline 141 & $\mathrm{EH}$ & 139 & 1392 & $04.23 / 1391.37$ & irr & $Q$ & es & $\mathrm{g}$ & $\mathrm{CPr}$ & pastillas repujadas \\
\hline 142 & $\mathrm{EH}$ & 139 & 1393 & $04.23 / 1391.37$ & irr & Q & es & $\mathrm{g}$ & $\mathrm{CPr}$ & pastillas repujadas \\
\hline 143 & $\mathrm{EH}$ & 139 & 1394 & $04.23 / 1391.37$ & irr & Q & es & $\mathrm{g}$ & $\mathrm{CPr}$ & pastillas repujadas \\
\hline 144 & $\mathrm{EH}$ & 139 & 1395 & $04.23 / 1391.37$ & irr & $\mathrm{Q}$ & es & $\mathrm{g}$ & $\mathrm{CPr}$ & pastillas repujadas \\
\hline 145 & $\mathrm{EH}$ & 126 & 1261 & $04.23 / 1261,1$ & red & $\mathrm{Q}$ & mo & $f / g$ & $\mathrm{CPr}$ & \\
\hline 146 & EP & 15 & 155 & $2009 / 38 / 155 / 2$ & red & $\mathrm{Q}$ & es & $\mathrm{m}$ & $\mathrm{CPr}$ & acanalada \\
\hline 147 & EP & 14 & 141 & $2009 / 38 / 141 / 28$ & irr & $\mathrm{L}$ & es & $\mathrm{m}$ & $\mathrm{CPr}$ & \\
\hline 148 & EP & 3 & 31 & $2009 / 38 / 031 / 1$ & irr & $Q$ & mo & $\mathrm{g}$ & $\mathrm{CPr}$ & pastillas repujadas \\
\hline 149 & EP & 5 & 51 & $2009 / 38 / 051 / 7$ & red & $\mathrm{Q}$ & es & $\mathrm{m}$ & $\mathrm{CPr}$ & moldura \\
\hline 150 & EP & 8 & 81 & $2009 / 38 / 051 / 8$ & red & $\mathrm{L} / \mathrm{Q}$ & $\mathrm{mo}$ & $\mathrm{f} / \mathrm{m}$ & $\mathrm{CPr}$ & perforaciones \\
\hline
\end{tabular}


Túmulo IL.C1 de Cótar/ Páramo de Rebollo I (ILC), Mojabarbas (MB), Villafría V (VFV), La Ampolilla (LA), La Tejera (LT) y Carrera Vega $(\mathrm{CV})$. El conjunto se configuraba con piezas con atribuciones del Calcolítico Inicial (Precampaniforme, $\mathrm{CPr}$ ) y Calcolítico Final (Campaniforme, CC). Se recogieron fragmentos evitando, en la medida de lo posible, la incorporación de grandes inclusiones y de concreciones que alterasen el análisis.

La preparación de las muestras fue llevada a cabo en el Área de Química Analítica de la Universidad de Burgos siguiendo los siguientes pasos:

1. Pulverización y homogenización de cada muestra en un mortero de ágata.

2. Toma de muestra para el análisis $(0.0100 \pm 0.0001 \mathrm{~g})$.

3. Disolución de parte de la muestra con una cantidad conocida de la mezcla de ácidos.

4. Extracción y dilución de la cerámica disuelta.

En la etapa de disolución, la elección de un método específico dependerá en gran medida de la técnica que se vaya a utilizar para la determinación de los metales y del problema arqueológico a resolver. En este caso, el análisis se llevó a cabo con un Espectrómetro de Masas con sistema Plasma Acoplado Inductivamente (ICP-MS) situado en el centro de I+D+I de la Universidad de Burgos. Con esta técnica se pueden alcanzar límites de detección del orden de las ppb (partes por billón) incluso en el análisis semicuantitativo, el cual se lleva a cabo con una calibración interna del equipo.

Las muestras se disuelven parcialmente con un tratamiento ácido secuencial, tratamiento muy habitual en este tipo de muestras (9-11). En primer lugar se añaden 10 microlitros de ácido fluorhídrico (HF) para eliminar parte del silicio de la muestra. Cuando se observa que desaparecen los vapores de $\mathrm{SiF}_{4}$, se añaden 100 microlitros de ácido nítrico (HNO3), agitando cuidadosamente la mezcla resultante. Todo este proceso se realiza en una campana extractora de gases. Se deja reaccionar durante 24 horas y se diluye la muestra 10 veces con agua desionizada calidad miliQ (Millipore). Tras eliminar el residuo sólido resultante, se diluye nuevamente la muestra 10 veces y se acidifica con $\mathrm{HNO}_{3}$.
Aunque no se disuelve toda la muestra, el hecho de realizar un tratamiento idéntico en cada una de ellas, permite obtener un extracto de iones metálicos comparable para cada caso. Hay que tener en cuenta que los datos que posteriormente trataremos estadísticamente, son las cuentas relativas obtenidas a partir de los datos que proporciona el equipo. El valor relativo se obtiene dividiendo el número de cuentas medido para cada elemento entre el número de cuentas totales obtenido para todos los elementos considerados $(\mathrm{Li}$, $\mathrm{Na}, \mathrm{Mg}, \mathrm{Al}, \mathrm{Ca}, \mathrm{Ti}, \mathrm{V}, \mathrm{Cr}, \mathrm{Mn}, \mathrm{Fe}, \mathrm{Co}, \mathrm{Ni}, \mathrm{Cu}, \mathrm{Zn}, \mathrm{Ga}, \mathrm{Se}, \mathrm{Sr}, \mathrm{Y}$, In, $\mathrm{Sn}, \mathrm{Ba}, \mathrm{La}, \mathrm{Ce}, \mathrm{Nd}, \mathrm{W}, \mathrm{Pb}$ y Bi). De esta forma, se obtiene la proporción relativa de metales en cada disolución para las todas las muestras.

\section{RESULTADOS}

Los resultados obtenidos (tabla 2) muestran las cuentas relativas para cada muestra, mostrando diferencias que pueden resultar significativas (12) en cuanto a la composición química. Para poder realizar cualquier tipo de interpretación de los datos se debe comprobar si estas diferencias tienen su traducción en lenguaje matemático y en qué sentido. Para ello se ha empleado el tratamiento estadístico.

\section{ANÁLISIS ESTADÍSTICO}

El análisis estadístico se ha efectuado con la aplicación Statgraphics Centurion ${ }^{\circledR}$ a través de varias técnicas exploratorias distintas.

En primer lugar, se ha llevado a cabo un Análisis de Componentes Principales (ACP), cuyo propósito es obtener un número reducido de combinaciones lineales de las 27 variables que expliquen la mayor variabilidad en los datos estandarizados. En este caso, 7 componentes tuvieron eigenvalues mayores o iguales que 1,0. En conjunto, se explica un $79,05 \%$ de la variabilidad de los datos originales. La representación gráfica de los scores o residuos de las nuevas variables (figura 3) manifiesta una distribución de los casos en la que se aprecia la existencia de un gran grupo de piezas

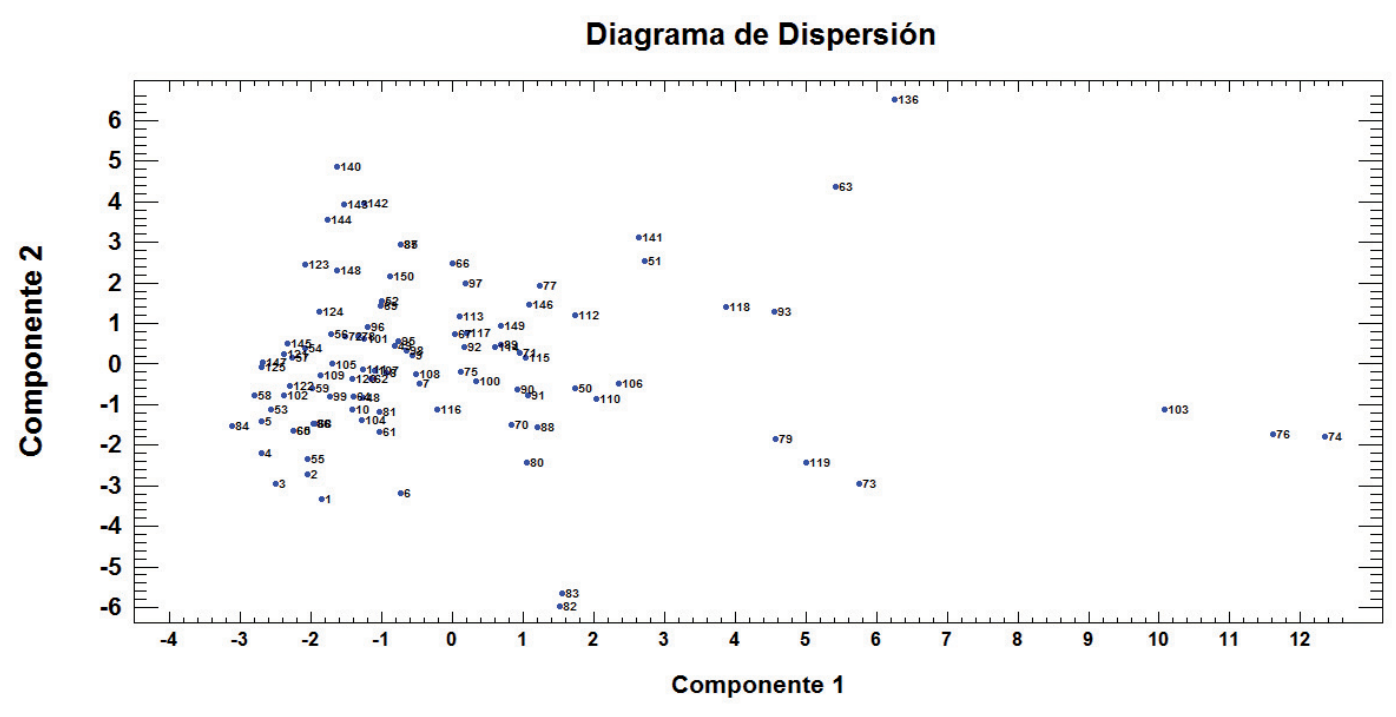

Figura 3. Gráfico representativo del Análisis de Componentes Principales 


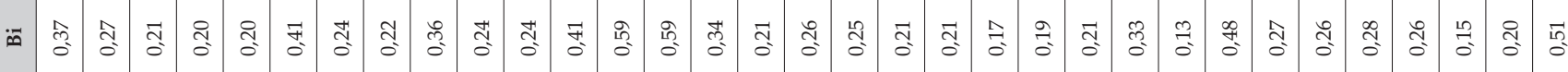

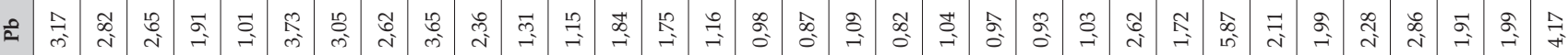

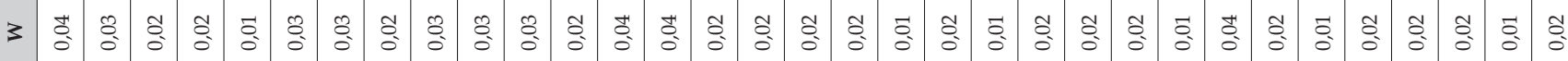

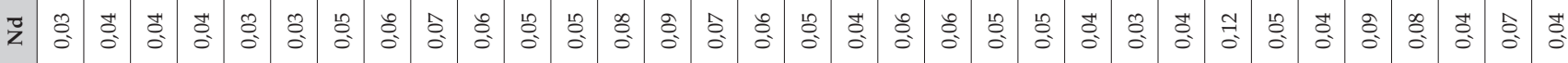

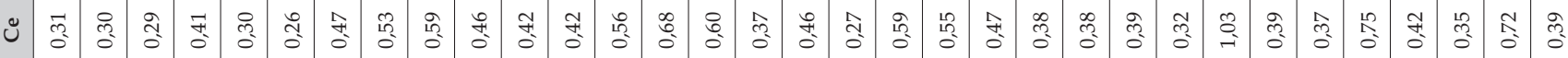

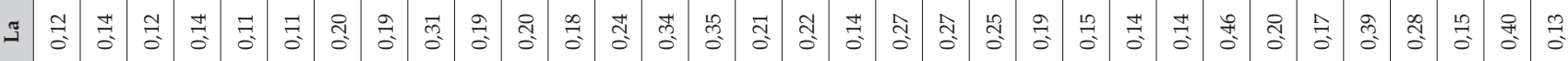

๓

可 क

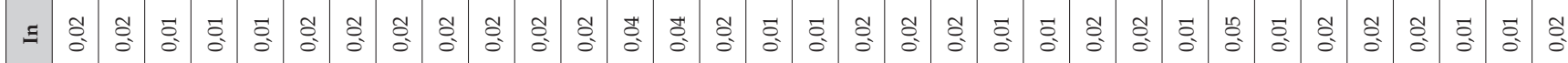

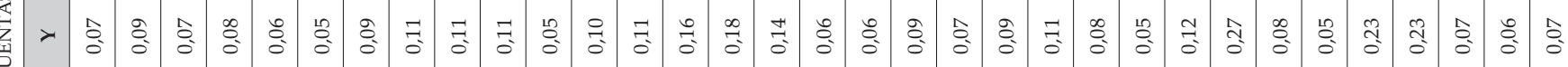

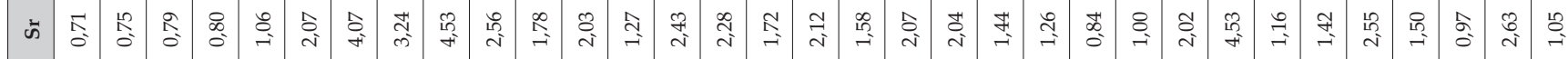

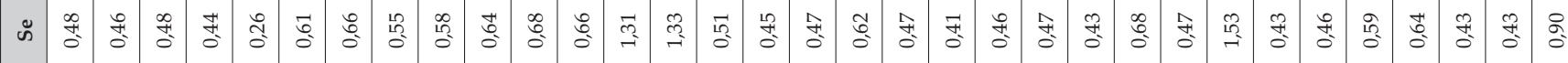

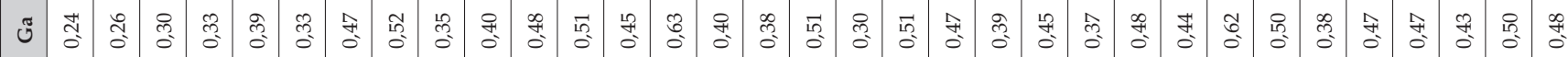

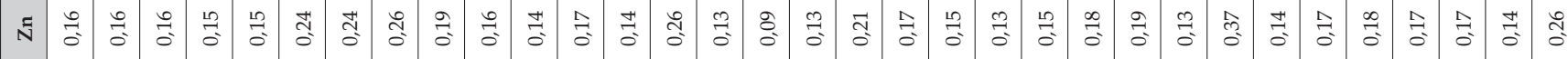

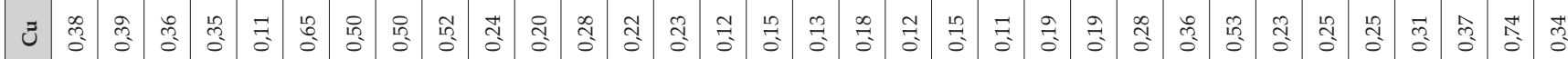

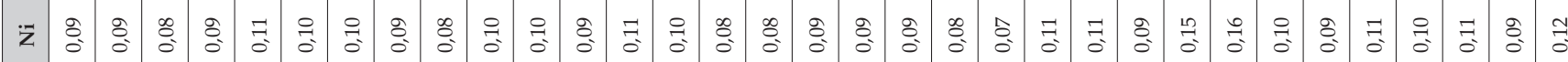

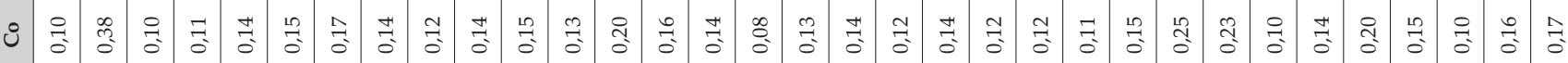

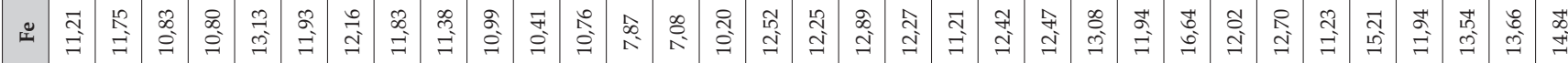



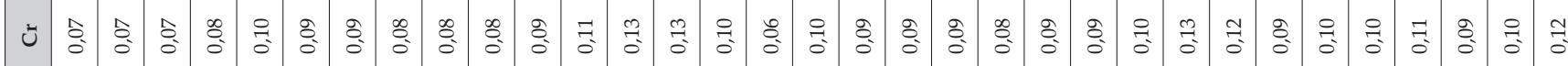

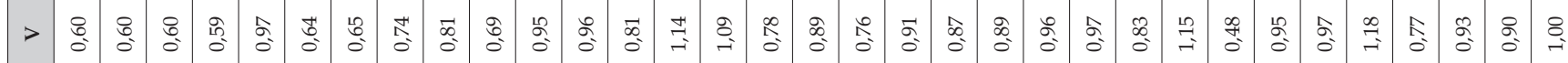

$F$ F $\begin{gathered}0 \\ \delta\end{gathered}$

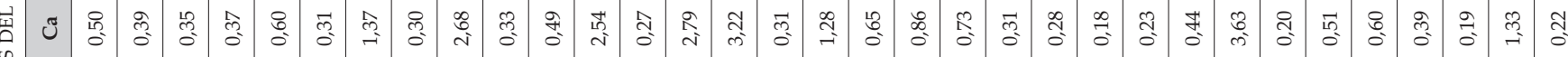

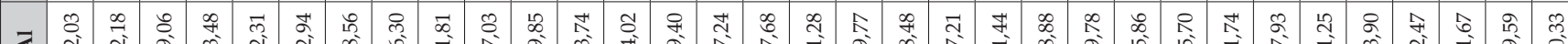

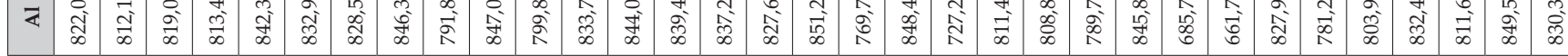

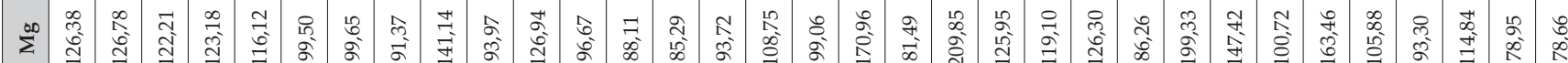

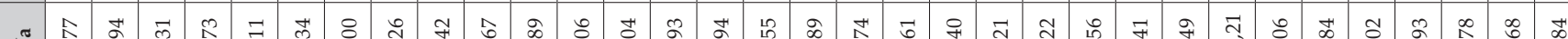

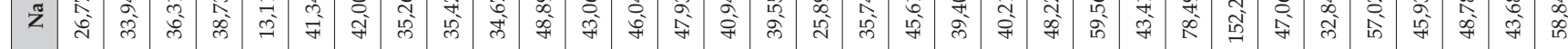

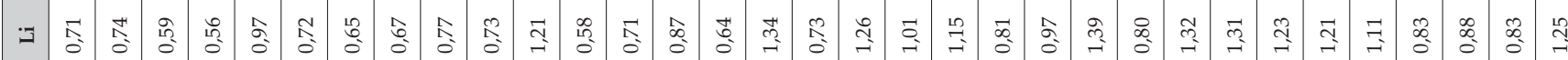




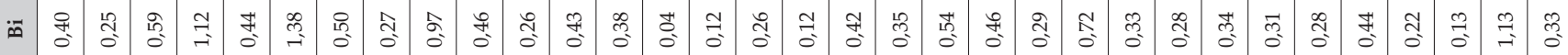

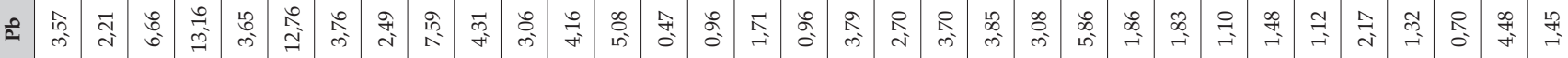

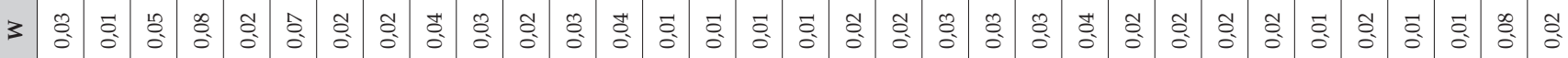

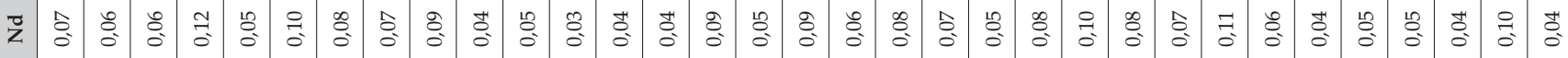

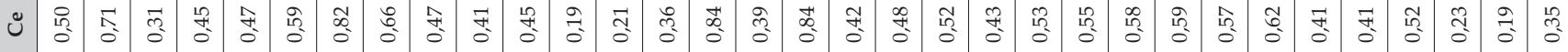

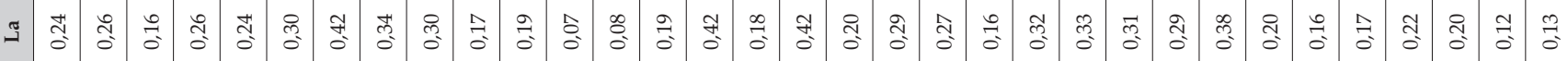
m क $\begin{aligned} & \Delta \\ & 0\end{aligned}$

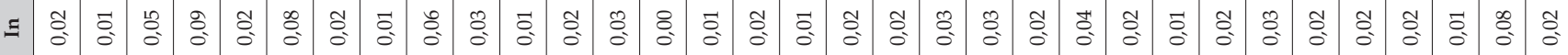

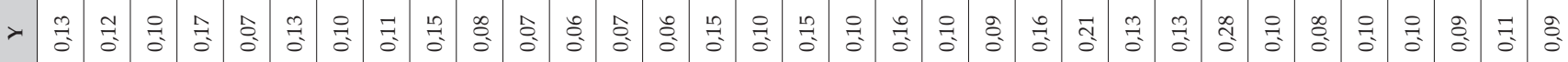

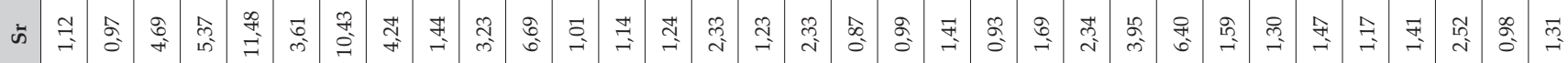

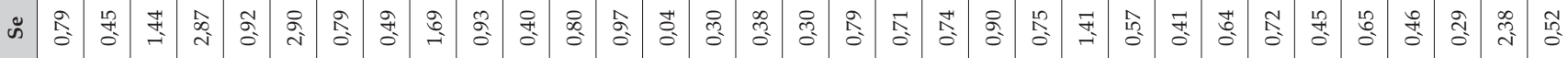

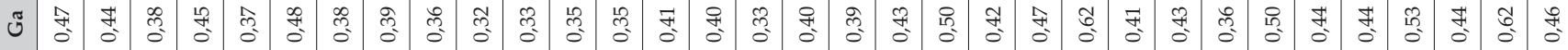

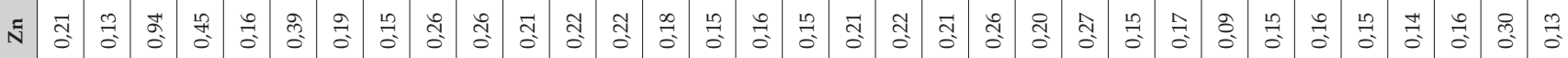

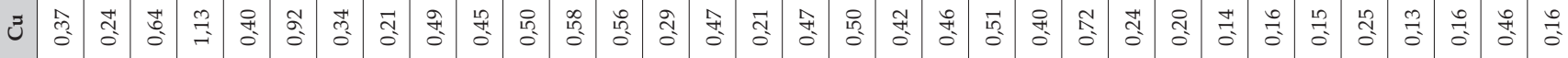

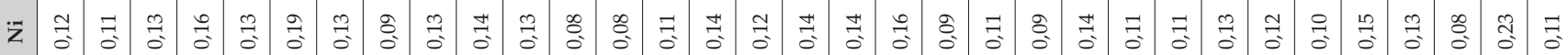

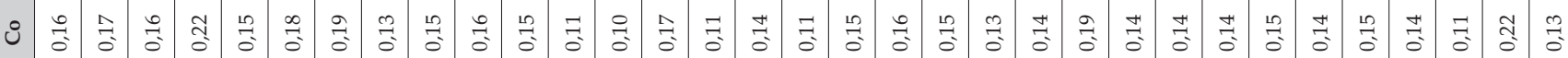

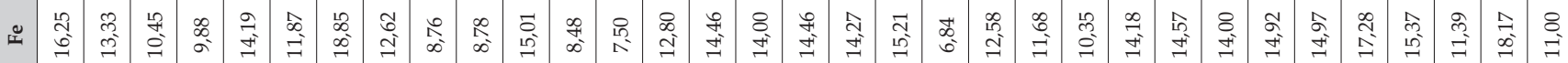



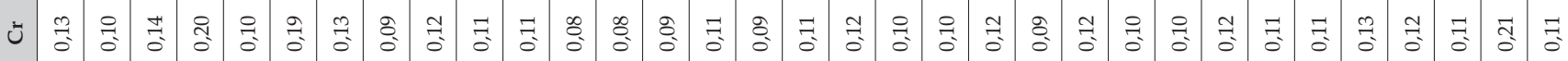

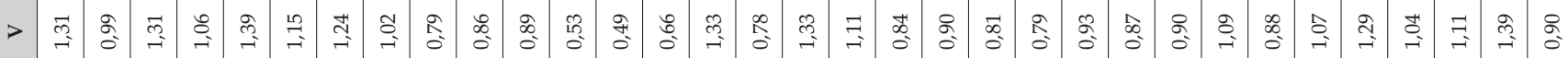

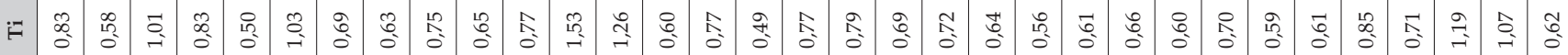



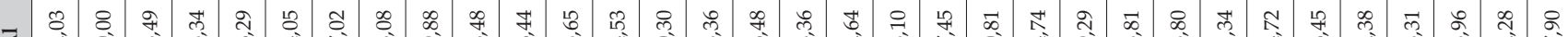

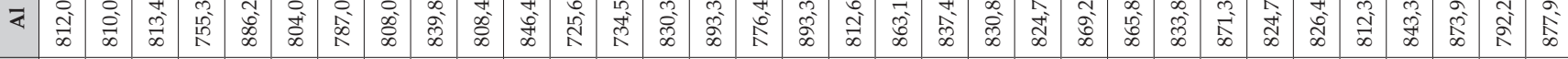

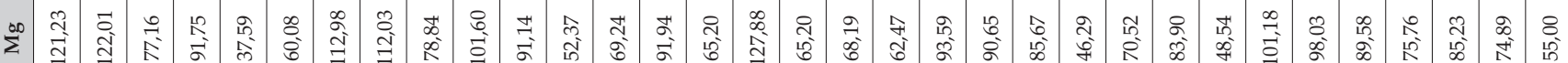
$\approx$ \&

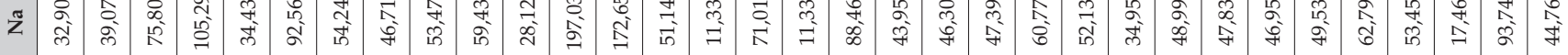

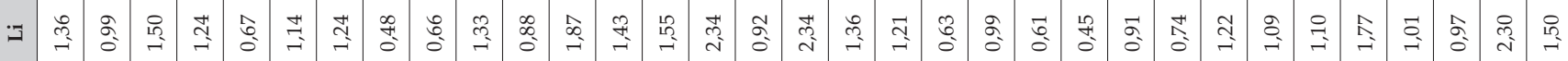

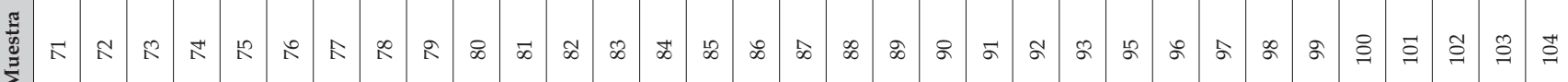




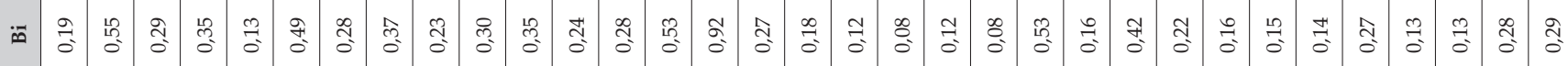

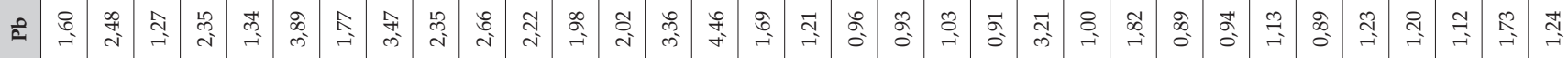

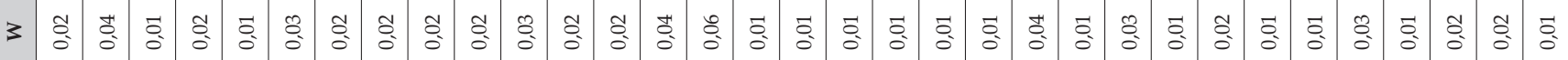
z z

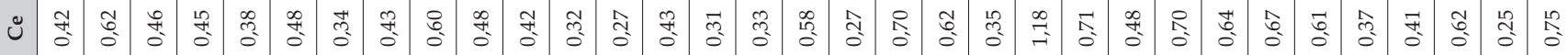


๓

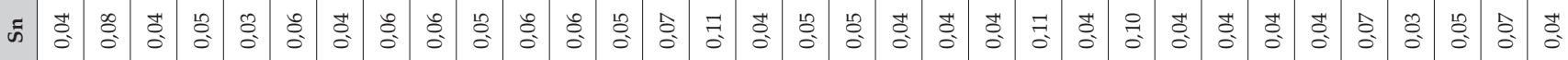

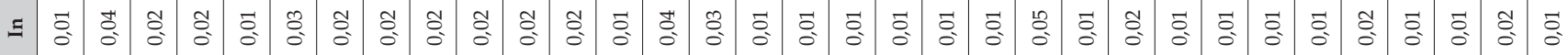

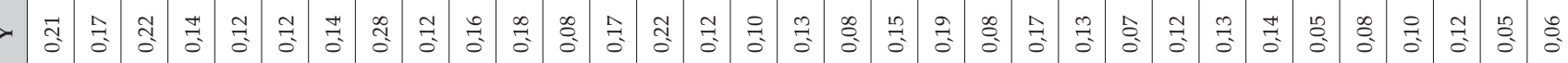

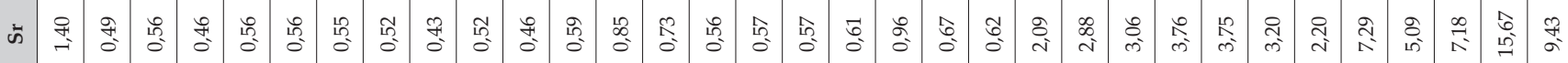
๓ $\begin{gathered}0 \\ \delta\end{gathered}$ I 舟 J



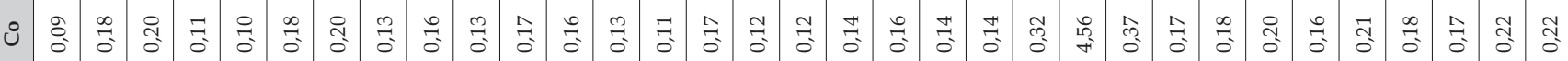

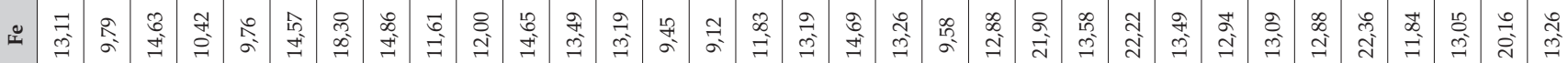

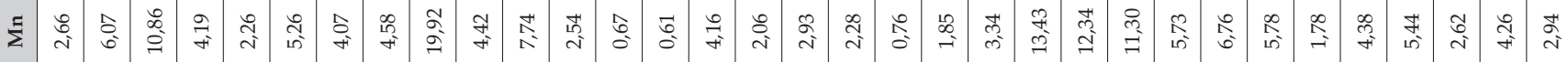

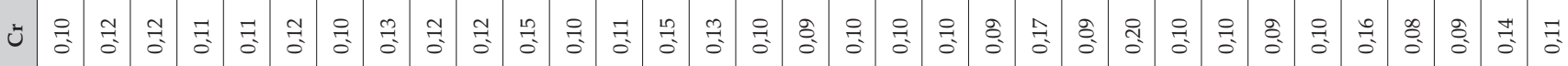

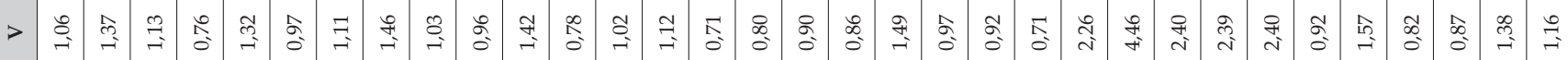

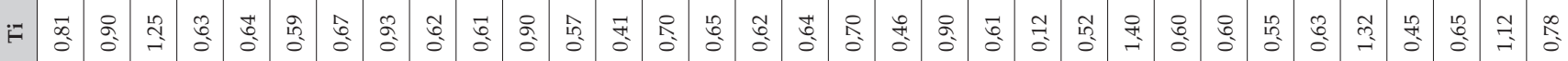

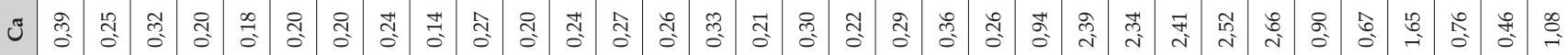

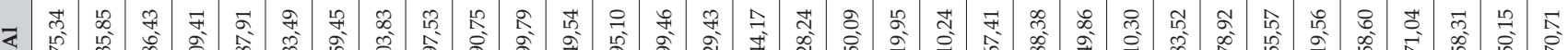

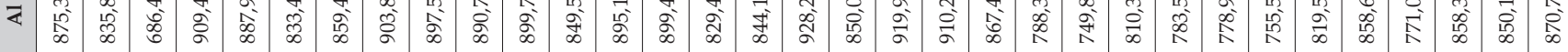

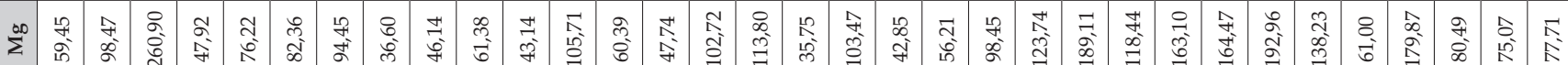
$\approx$ z

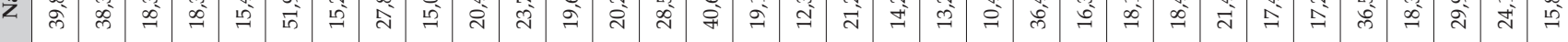








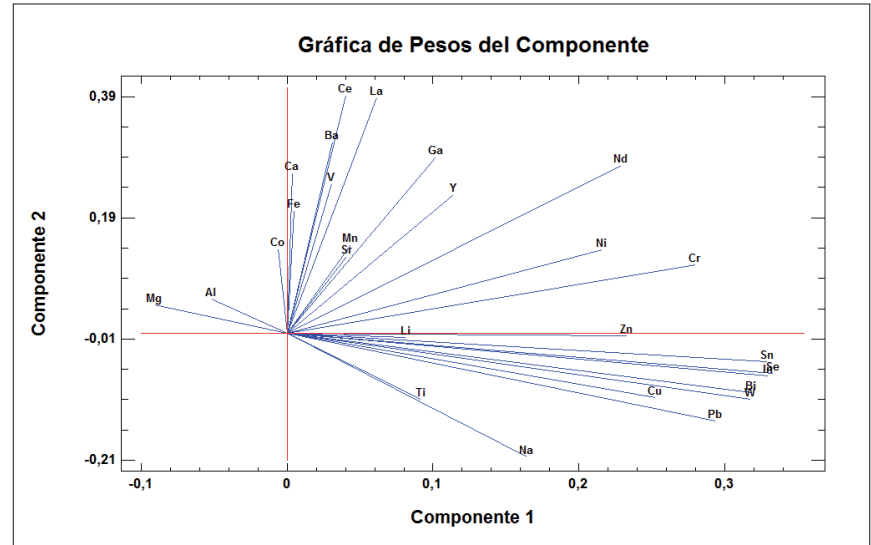

Figura 4. Proyección de las variables con respecto a los componentes 1 y 2.

con características químicas similares que podemos considerar como principal o mayoritario, del cual se separan claramente algunas muestras $(63,73,74,76,79,82,83,103,119$ y 136). La observación más detallada permite reconocer otros grupos menos claros que se ubican de manera periférica al gran grupo principal: un segundo grupo formado por 85, 122, 140, 141, 142, 144 y 148; agrupadas por pares las muestras 51, 141, 93 y 118.

En la figura 4 se representa la contribución de cada ion metálico en las nuevas variables. La distribución se explica en algunos casos por la influencia de determinados iones metálicos como el Na respecto a 82 y 83 ; el $\mathrm{Pb}$ respecto a 73, 79 y 119; o Nd en relación a 63 y 136. En este sentido, las muestras 74, 76 y 103 se ven afectadas por la mayor presencia de Sn, $\mathrm{Se}, \mathrm{In}, \mathrm{Bi} \mathrm{W}$ y $\mathrm{Pb}$. También es notable el peso de Ca dentro de la composición del conjunto formado por 85, 122, 140, 141, 142, 143, 144, 148 y 150, y otras muestras como 7, 51, 69 y 147, aunque esta variable se ve compensada por el peso de otras en el análisis.

El ACP se complementó con la realización de un Análisis Cluster con el objeto de observar y especificar de forma más detallada la relación de las agrupaciones y facilitar, posteriormente, la explicación de su significación. Para la agrupación jerárquica se empleó el método de promedio de grupo, empleando la distancia euclídea al cuadrado y estandarizándo los datos. A pesar de pequeñas diferencias, el Análisis Cluster parece reafirmar lo que se desprende del ACP (figura 5). Bien es cierto que aunque se pueden observar algunas agrupaciones internas que se corresponden con las observaciones del ACP, es muy difícil encontrar discrepancias entre los casos que sean significativas a la hora de separar claramente grupos. Esta particularidad solo se observa de nuevo con algunas muestras situadas como casos anómalos (outliers) en el extremo del gráfico, las cuales vienen a coincidir con los casos que claramente se separaban en el ACP.

Por lo tanto, el análisis manifiesta que existe un gran grupo de muestras con unas características químicas semejantes (Conjunto A), con algunas leves diferencias internas. El grado de homogeneidad de la mayoría de los materiales permite considerarlos pertenecientes a un mismo grupo. La falta de diferencias que permitiría establecer un origen diverso (13), determina que se pueda contemplar como hipótesis que la mayoría de las cerámicas analizadas pueden tener una procedencia afín. El análisis parece indicar que dentro del Conjunto A se pueden reconocer subconjuntos diferentes, al menos 4. Esta posibilidad ha sido explorada a través de un análisis $\mathrm{K}$-medias, tomando una $\mathrm{K}=5$, para poder discriminar las muestras atípicas como grupo independiente, y analizando los datos sin estandarizar. Como referencia se han utilizado $\mathrm{Fe}$ y Al que son algunos de los elementos mayoritarios en cerámicas, que su vez provienen de arcillas donde, por ejemplo, Si y Al suelen ser mayoritarios. En este caso el test separa los casos en grupos distintos cuyo fundamento parece tener algún tipo de relación con la atribución (figura 6). Al menos eso se desprende observando, por ejemplo, el conglomerado 5 en el que todas las muestras excepto 1 muestran atribuciones campaniformes. Algo semejante sucede con el conglomerado 2 pero en sentido contrario: todas las muestras menos 1 tienen una atribución Precampaniforme. Los conglomerados intermedios comparten piezas de ambas atribuciones. Esta apreciación parece reflejar algún tipo de vínculo entre las características químicas de las cerámicas y la elaboración de las mismas. El tipo de análisis efectuado no permite valorar esta relación con mayor profundidad. No obstante, este rasgo parece indicar alguna diferencia



Figura 5. Dendrograma que refleja la ordenación de las muestras según el Análisis Cluster 


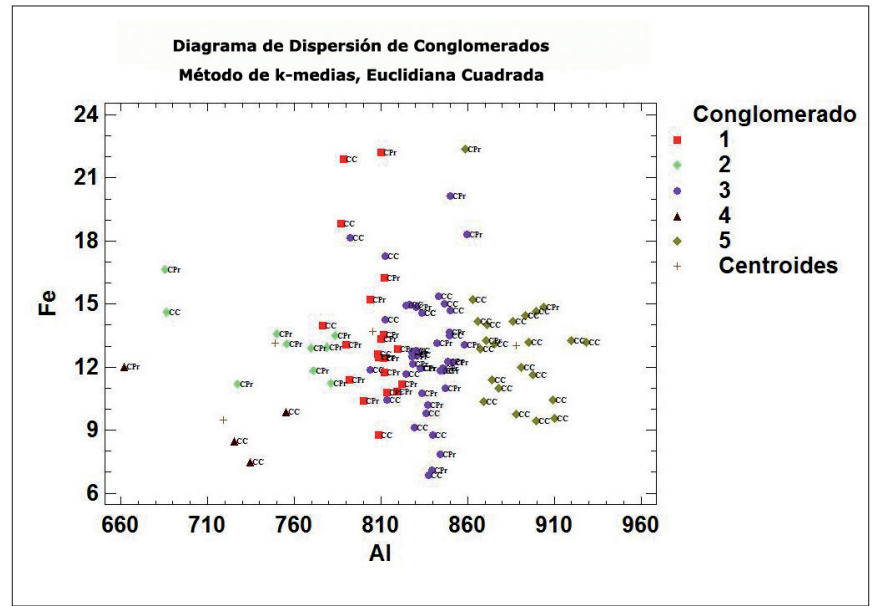

Figura 6. Representación gráfica del Análisis K-medias. Clasificación tipológica de las muestras: Calcolítico Precampaniforme (CPr), Calcolítico Campaniforme ciempozuelos (CCc), Calcolítico Campaniforme internacional (CCi), Calcolítico Campaniforme puntillado (CCp), Calcolítico Campaniforme Valle del Ebro (CCe), Calcolítico Campaniforme liso $(\mathrm{CCl})$. $\mathrm{Al}$, Fe y $\mathrm{Ca}$ son algunos de los elementos mayoritarios de las cerámicas.

asociada a la cronología. Como hipótesis se puede señalar que muy probablemente haya algún tipo de modificación en el proceso productivo. Esta puede relacionarse con una selección o modificación de las materias primas empleadas en la fabricación, pero para confirmar esta hipótesis serían necesarios otros análisis que suelen ser habituales (14-16), por ejemplo, DRX o lámina delgada.

\section{DISCUSIÓN}

Como se ha señalado anteriormente, el objetivo del estudio no era otro que comprobar la composición química de una muestra de cerámicas calcolíticas de la Cuenca Media del Arlanzón y verificar si los resultados obtenidos tenían alguna significación arqueológica. Nuestro interés se centra no tanto en la discusión de las características químicas de las piezas sino en la resolución de otras problemáticas vinculadas a estas cerámicas prehistóricas. En este sentido, el punto de partida lo establecen los datos morfo-tipológicos. El conjunto tiene los siguientes rasgos: de manera general se reconocen tanto cerámica precampaniforme como campaniforme, cuyos atributos son los habituales en la zona de estudio. Dentro de este conjunto se aprecian ciertas piezas particulares con motivos decorativos campaniformes, ya sean internacionales o relacionados con el valle del Ebro. Teniendo esto en cuenta se podría establecer una discusión sobre la procedencia local o foránea de la cerámica.

En este sentido, para que las desigualdades sean significativas con el fin de segregar entre cerámicas locales y no locales se necesitan rangos mayores que los detectados dentro del grupo mayoritario, como demuestra la bibliografía al respecto (14-24). La lectura en términos arqueológicos de esta afirmación se traduce en la consideración de que este gran grupo se puede haber fabricado con una materia prima semejante, ya sea por ser una materia prima similar o cuyas condiciones se han homogeneizado artificialmente. En resumidas cuentas se puede considerar con un elevado grado de probabilidad que este gran grupo puede tener una manufactura local. Este hecho no es sorprendente dado que es una tendencia generalizada en los conjuntos cerámicos de la Prehistoria europea, tanto campaniformes $(1,25-30)$ como de otras atribuciones $(8,25,31)$. Como hecho relevante cabe señalar la pertenencia a este grupo aparentemente local de las piezas con motivos decorativos propios del valle del Ebro en el yacimiento de Villafría V. Por lo tanto, si las piezas no viajaron, ¿qué viajó? ¿Los motivos, las personas?

Fuera de este conjunto únicamente quedarían las piezas que el análisis estadístico señala como valores anómalos (outliers). En un procedimiento estadístico habitual estas muestras se considerarían aberrantes, pudiendo incluso descartarse de la investigación debido a la potencial distorsión que provocan. No obstante se debe tener en cuenta la particularidad de la muestra con la que se trabaja: un conjunto de cerámicas prehistóricas fabricadas a mano dentro de un contexto doméstico de producción $(1,8,25)$ y cuya circulación es mínima. Desde nuestro punto de vista, estas piezas singulares sí tienen significación arqueológica.

Curiosamente, este grupo de piezas atípicas está dominado por cerámicas de tipo campaniforme internacional y que proceden de yacimientos distintos: Túmulo IL.C1 de Cótar/ Páramo de Rebollo I (muestras 82 y 83), Castillo de Burgos (muestras 73, 74, 76 y 79); a las que se añaden algunas piezas de estilo Ciempozuelos recogidas en La Tejera (muestra 119) y Cuesta Vega (muestra 136); y una única pieza precampaniforme de El Hornazo (muestra 63). La particularidad de estas muestras es mayor si se tiene en cuenta que otras procedentes de los mismos yacimientos e, incluso, de los mismos contextos, no difieren químicamente de las del Conjunto A. Esto, desde nuestro punto de vista, descarta algunas explicaciones plausibles ligadas a transformaciones originadas por procesos postdeposicionales que pudieran haber alterado químicamente las piezas. Otra explicación plausible es que estas piezas pudieran proceder de vetas de arcilla distintas. No podemos desechar esta hipótesis con rotundidad pero dudamos de ella. Es bastante improbable que no se viesen más casos al respecto, teniendo en cuenta la extensión de la zona estudiada $\left(699 \mathrm{~km}^{2}\right)$. A ello se suma el contexto de producción: doméstico, muy flexible, que aprovecha cualquier veta a su alcance para satisfacer unas necesidades básicas, muy vinculadas a la supervivencia, de producción y consumo. En tales circunstancias cada yacimiento (305 en la zona de estudio) se convierte en un potencial centro de producción en el que se aprovechan vetas diversas. Por esta razón, la búsqueda de fuentes concretas se convierte en algo prácticamente imposible. En todo caso, sí que creemos que la utilización de diferentes vetas o tipos de materia prima se puede relacionar con la existencia de subgrupos dentro del Conjunto A.

Para finalizar es necesario señalar que la distribución de las muestras por yacimientos o contextos no tiene una traducción clara en el estudio planteado. La separación en función de las distintas entidades arqueológicas sería una situación esperable debido a la utilización de vetas distintas y a la acreditada afección postdeposicional de muchas de las piezas en forma de concreciones que podrían haber proporcionado sesgos reforzando una distribución por contextos. La estructura de los datos obtenida nos hace suponer que no existen diferencias muy significativas desde el punto de vista químico en la materia prima utilizada. Esto enfatiza una procedencia semejante de las piezas dentro de un entorno con arcillas con características químicas similares. 


\section{CONCLUSIONES}

Por lo tanto, los datos permiten asumir con una alta probabilidad que la mayoría de las piezas se han fabricado en la Cuenca Media del Arlanzón, con variaciones técnicas en el proceso productivo que dejan huella sobre todo a finales del ciclo estudiado (campaniforme). Por otro lado, existe un grupo minoritario no fabricado en la Cuenca Media del Arlanzón cuyo número (9 de 99) supone que 9, $09 \%$ de las cerámicas pueden ser de origen foráneo. Si atendemos los casos en virtud de su atribución cultural el porcentaje oscila entre 2,08\% del Calcolítico Inicial (Precampaniforme) y 15,68\% del Calcolítico Final (Campaniforme). Este último porcentaje viene a coincidir con el detectado en el estudio de la cerámica campaniforme del Guadiana, que estima en un $14 \%$ el total de cerámica intercambiada (32). En aquel espacio también destaca el campaniforme internacional como elemento más intercambiado: un $8 \%$ de las piezas no locales frente al $2 \%$ en el resto de estilos, lo que viene a coincidir con lo reconocido en el Calcolítico Inicial (Precampaniforme) del Arlanzón.

De estos datos se pueden extraer una serie de lecturas. Las analíticas realizadas sobre los extractos manifiestan que la producción de cerámica durante el III milenio cal B.C. en la Cuenca Media del Arlanzón se realiza bajos unas condiciones de producción que tienden a abastecerse de materia prima local. Esto encaja con los presupuestos de partida. Ahora bien, también se reconoce la incorporación de objetos, ya sea como bienes en sí mismos ya sea por sus contenidos, o materias primas alóctonas, sobre todo en el Calcolítico Final (Campaniforme). Mientras los atributos morfo-tipológicos de las piezas precampaniformes no revelan información relacionable a la procedencia local o foránea, las muestras campaniformes presentan un patrón interesante. Los objetos reconocidos como foráneos incorporan mayoritariamente motivos vinculados al Campaniforme Internacional. De ello se puede deducir que al menos una parte de la vajilla de este tipo no tiene su origen en la Cuenca Media del Arlanzón. Por lo tanto, son elementos alóctonos. El alcance de estas observaciones deber ser valorado convenientemente.

En este sentido, hemos de recordar que Salanova (33) señala la difusión de los elementos campaniformes mediante tres modos: 1) a través del movimiento de "artesanos"; 2) a través de las imitaciones de las poblaciones indígenas y, por último, 3) a través de los intercambios de recipientes. No parece percibirse, a tenor de los datos, una interpretación unívoca para la Cuenca Media del Arlanzón sino más bien una combinación de distintas vías para explicar la incorporación de cerámica campaniforme. En definitiva, se podría relacionar con un modelo de producción e intercambio que se basa en la producción doméstica, de autosubsistencia, que se combina con una reducida cantidad de elementos foráneos. Esta aseveración ha de considerarse como una hipótesis que ha de ser contrastada con nuevos estudios en el futuro.

\section{AGRADECIMIENTOS}

Queremos expresar nuestro agradecimiento a las empresas Antequem Arqueología y Medio Ambiente S.L., 2B Arqueología y Gestión del Patrimonio S.L. y a D. Jesús M. Martínez González por habernos proporcionado los materiales arqueológicos para el estudio. Este trabajo ha sido posible gracias al Proyecto "Introducción al estudio interdisciplinar de las sociedades segmentarias en el centro-norte peninsular (BU009A09)", al Fondo Social Europeo y a la Junta de Castilla y León.

\section{BIBLIOGRAFÍA}

1. Carmona Ballestero, E. (2010), Prestigio y emulación en espacios marginales: la cerámica campaniforme de Paulejas (Quintanilla del Agua, Burgos), Universidad de Burgos, Burgos.

2. Carmona Ballestero, E. (2013): El calcolítico en la Cuenca Media del río Arlanzón (Burgos, España). Comunidades campesinas, procesos históricos y transformaciones, BAR International Series., Archaeopress, Oxford.

3. Carmona Ballestero, E., Delgado Arceo, M.E, Villanueva Martín, L. (2013): "El yacimiento de El Púlpito (Villalonquéjar, Burgos). Nuevos datos sobre el Calcolítico en la Cuenca Media del Arlanzón". En Sastre Blanco, J.C. Catalán Ramos, R., Fuentes Melgar, P. (coords.): Arqueología en el Valle del Duero. Del Neolítico a la Antigüedad tardía: nuevas perspectivas. La Ergástula: 19-32

4. Alameda Cuenca-Romero, M.A., Carmona Ballestero, E., Pascual Blanco, S. Martínez Diez, G., Díez Pastor, C. (2011): “El “Campo de Hoyos” calcolítico de Fuente Celada (Burgos): datos preliminares y perspectivas", Complutum 22 (1): 195-206

5. Orton, C., Tyers, P., Vince, A. (1997): La cerámica en arqueología. Crítica, Barcelona.

6. Calvo Trias, M., Fornés Bisquerra, J., García Roselló, J., Guerrero Ayuso, V. M., Juncosa Vecchierini, E., Quintana Abraham, C. y Salvà Simonet, B. (2004): La cerámica prehistórica a mano: una propuesta para su estudio. El Tall, Mallorca.

7. Eiroa, J. J., Bachiller Gil, J. A., Castro Pérez, L., Lomba Maraundi, J. (1999): Nociones de tecnología y tipología en Prehistoria. Ariel, Barcelona.

8. Vega Maeso, C (2012): “The ceramics of El Mirón Cave: production, morphology and discard". En Straus, L. G. y González Morales, M. R. (eds.), El Mirón Cave, Cantabrian Spain. The site and Its Holocene archaeological record. University of New Mexico Press, Alburquerque:. 372-425

9. Hall, M. E., Brimmer, S. P., Li, F. H., Yablonsky L. (1998). “ICP-MS and ICPOES Studies of Gold from a Late Sarmatian Burial", Journal of Archaeological Science 25:545-552.

10. Hart, F. A., Adams S. J. (1983), “The Chemical Analysis of Romano-British Pottery from the Alice Holt Forest, Hampshire, by means of Inductivelycoupled Plasma Emission Spectrometry", Archaeometry 25 (2):179-185.

11. Aldrabee, A., Wriekat, A-H. (2011), “Archaeometric characterization of ancient glazed pottery sherds from Khirbet Faris, Jordan by inductively coupled plasma mass spectrometry (ICP-MS)", Microchemical Journal 99: 289-295

12. Baxter, M.J. (1994), Exploratory Multivariate Analysis in Archaeology, Edinburgh University Press.

13. Fernandes, F., Jordán, M. M., Martín, J. D., Sanfeliu, T., Clausell, G. (1997), "Estudio arqueométrico de cerámicas ibéricas del yacimiento del Torrelló de Almassora (Castellón)". II Congreso Nacional de Arqueometría, Caesaraugusta, 73, 99-107.

14. González García, F., González Rodríguez, M., González Vilchez, M. C., Vallejo Triano, A. (1992): "Estudio arqueométrico de algunas cerámicas medievales de Madinat Al-Zahra (Córdoba)", Bol. Soc. Esp. Ceram. Vidr. 31 (6): 491-98.

15. García Heras, M. (1994): “Estudio arqueométrico de la cerámica de Izana (Soria) y de otros yacimientos celtibéricos del Alto Duero", Bol. Soc. Esp. Ceram. Vidr. 33 (6): 315-25.

16. Igeal, J., Pérez-Aranteguiz, J., Lapuente, P., Saiz, M. E., Burillo, F. (2013): "Producciones de cerámica Celtibérica procedentes del sistema Ibérico Central (España): Caracterización química y petrográfica", Bol. Soc. Esp. Ceram. Vidr.. 52 (1): 1-12.

17. Capel, J., Molina, F., Guardiola, E., Jiménez Cisneros, C. (1999) "Identificación de la procedencia de materiales cerámicos mediante la técnica de Espectrometría de Masas de Isótopos Ligeros Estables y estudio petrológico en Lámina Delgada", en J. Capel Martínez (ed.), Arqueometría y Arqueología, Colección monográfica Arte y Arqueología, Universidad de Granada, Granada, 89-105,

18. J. Capel, F. Molina, T. Nájera, J. Linares, F. Huertas (2001), “Aproximación al estudio de procedencia y fabricación de las cerámicas campaniformes del yacimiento de la Edad del Cobre de Los Millares", en B. Gómez Tubío, M. A. Respaldiza, M. L. Pardo Rodríguez (eds.), III Congreso Nacional de Arqueometría, Universidad de Sevilla, Sevilla,: 207-217.

19. Estévez Morales, J. A. (1997), “Caracterización de cerámicas comunes romanas de Extremadura" II Congreso Nacional de Arqueometría, Caesaraugusta, 73, 67-76,

20. García Heras, M., Fernández Ruiz, R. (1997), "Primeros resultados de la caracterización arqueométrica de la cerámica numantina del siglo I a.c." , II Congreso Nacional de Arqueometrí, Caesaraugusta, 73, 59-65. 
21. García Heras, M. Fernández Ruiz, R., Tornero, J. D. (1999), “Análisis de cerámicas arqueológicas mediante Fluorescencia de Rayos X por Reflexión Total y contrastación mediante Análisis por Activación de Neutrones", en J. Capel Martínez (ed.), Arqueometría y Arqueología, Colección Monográfica Arte y Arqueología, Universidad de Granada, Granada, 173-85.

22. García Heras, M., Arenas Esteban, J. A., González Vilchez, M. C., González Rodríguez, M. (1999), "La caracterización de los materiales cerámicos del yacimiento celtibérico de "El Palomar" (Aragoncillo, Guadalajara), una producción especializada", en J. Capel Martínez (ed.), Arqueometría y Arqueología, Colección Monográfica Arte y Arqueología, Universidad de Granada, Granada, 143-58.

23. Olaetxea, C. (2000), La tecnología cerámica en la protohistoria vasca, Munibe (Número Monográfico).

24. Shennan, S. (1992), Arqueología cuantitativa, Crítica, Barcelona

25. Clop, X. (2007), Materia prima, cerámica y sociedad. La gestión de los recursos minerales para manufacturar cerámicas del 3100 al 1500 ANE en el nordeste de la Península Ibérica, 1660. BAR International Series, Archaeopress, Oxford.

26. Convertini, F, Querré, G. (1998), “Apport des études céramologique en laboratoire à la connaissance du Campaniformes: résultats, bilan et perspectives", Bulletin de la Société Préhistorique Française. 95: 333-41.

27. Delibes de Castro, G., Herrán Martínez, J. I. (2007), La Prehistoria, Biblioteca Básica de Valladolid. Diputación de Valladolid, Valladolid.

28. Dias, M.I., Prudêncio, M.I, Prates, S., Gouveia, M.A., Valera, A.C. (2000), «Tecnologias de produção e proveniência de material-prima das cerâmicas campaniformes da Fraga da Pena (Fornos de Algodres - Portugal)». Actas do $3^{\circ}$ Congresso de Arqueologia Peninsular, 4 (Pré-História Recente da Península Ibérica), ADECAP:. 253-68

29. Martínez Cortizas, A., Lantes Suárez, O., Prieto Martínez, M. P. (2011) «Cerámica campaniforme del NW de la Península Ibérica. Indagando en sus materias primas, elecciones tecnológicas y procedencia», en Prieto Martínez, M. P. y Salanova, L. (eds.), Las comunidades campaniformes en Galicia. Cambios sociales en el III y II milenios BC en el NW de la Península Ibérica, Diputación de Pontevedra, Pontevedra: 309-32.

30. Vander Linden, M. (2007): «What linked the Bell Beakers in third millenium BC Europe?, Antiquity. 81: 343-52.

31. Clop, X. (2004), “La gestión de los recursos minerales durante la prehistoria reciente en el noreste de la Península Ibérica", Cypsela. 15: 171-86.

32. Odriozola Lloret, C., Hurtado Perez, V., Dias, M. I., y Valera, A.C. (2008): "Produçao e Consumo de Campaniformes no Vale Do Guadiana: Uma Perspectiva Ibérica", Apontamentos de Arqueologia e Património. 3: 45-52.

33. Salanova, L. (2000), "Mecanismes de diffusion des vases campaniformes: les liens franco-portugais", III Congreso de Arqueología Peninsular, ADECAP: 399-404.

Recibido: $17 / 01 / 2013$

Recibida versión corregida: 08/12/2013

Aceptado: 10/12/2013 